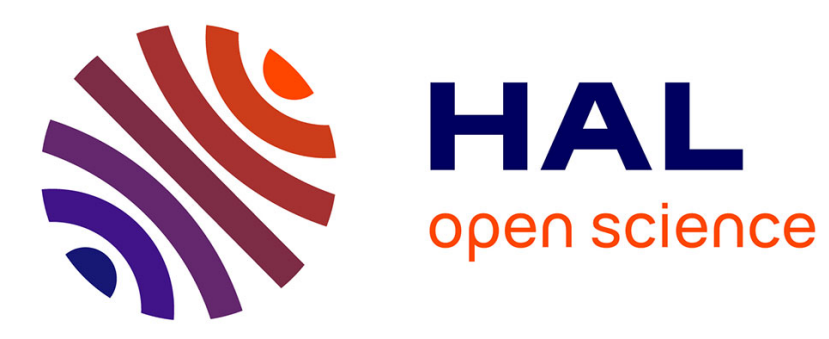

\title{
Skin absorption of mixed halide anions from concentrated aqueous solutions
}

\author{
Malgorzata Tarnowska, Yves Chevalier, Stéphanie Briançon, Claire Bordes, \\ Jacqueline Resende de Azevedo, Delphine Arquier, Thierry Pourcher, \\ Marie-Alexandrine Bolzinger
}

\section{To cite this version:}

Malgorzata Tarnowska, Yves Chevalier, Stéphanie Briançon, Claire Bordes, Jacqueline Resende de Azevedo, et al.. Skin absorption of mixed halide anions from concentrated aqueous solutions. European Journal of Pharmaceutical Sciences, 2021, pp.105985. 10.1016/j.ejps.2021.105985 . hal-03330438

\section{HAL Id: hal-03330438 \\ https://hal.science/hal-03330438}

Submitted on 31 Aug 2021

HAL is a multi-disciplinary open access archive for the deposit and dissemination of scientific research documents, whether they are published or not. The documents may come from teaching and research institutions in France or abroad, or from public or private research centers.
L'archive ouverte pluridisciplinaire HAL, est destinée au dépôt et à la diffusion de documents scientifiques de niveau recherche, publiés ou non, émanant des établissements d'enseignement et de recherche français ou étrangers, des laboratoires publics ou privés. 


\title{
Skin absorption of mixed halide anions from concentrated aqueous solutions
}

Małgorzata Tarnowska ${ }^{\mathrm{a}}$, Yves Chevalier ${ }^{\mathrm{a}}$, Stéphanie Briançon ${ }^{\mathrm{a}}$, Claire Bordes ${ }^{\mathrm{a}}$, Jacqueline Resende de Azevedo $^{\mathrm{a}}$, Delphine Arquier ${ }^{\mathrm{a}}$, Thierry Pourcher ${ }^{\mathrm{b}}$, Marie-Alexandrine Bolzinger ${ }^{\mathrm{a}}$

${ }^{a}$ Univ Lyon, Université Claude Bernard Lyon 1, CNRS UMR 5007, Laboratoire d'Automatique, de Génie des Procédés et de Génie Pharmaceutique (LAGEPP), 43 bd du 11 Novembre 1918, 69622, Villeurbanne, France.

${ }^{\mathrm{b}}$ Laboratory Transporter in Imaging and Radiotherapy in Oncology (TIRO), University Côte d'Azur, Institut de biosciences et biotechnologies d'Aix-Marseille (BIAM), Commissariat à l'Énergie Atomique, Faculté de Médecine, 28 av de Valombrose, Nice, France.

\begin{abstract}
Non-ideal behaviour of mixed ions is disclosed in skin absorption experiments of mixed halide anions in excised pig skin. Comparison of skin absorption of pure and mixed ions shows enhanced penetration of chaotropic ions from mixed solutions. An experimental design and statistical analysis using a Scheffé $\{3,2\}$ simplex-lattice allows investigating the full ternary diagram of anion mixtures of fluoride, bromide and iodide. Synergism in mixed absorption is observed for chaotropic bromide and iodide anions. A refined analysis highlighting specific interactions is made by considering the ratio of the absorbed amount to the ion activity instead of the directly measured absorbed amount. Statistical analysis discards non-significant effects and discloses specific interactions. Such interactions between bromide and iodide cause an absorption enhancement of their partner by a factor of 2-3 with respect to the case of ideal mixing. It is proposed that enhanced absorption from mixed solution involves the formation of neutral complex species of mixed bromide and iodide with endogenous magnesium or calcium inside stratum corneum.
\end{abstract}

Keywords: Skin absorption; Anions absorption; Ionic interactions; Hofmeister series; Halide ions; Experimental design 


\section{Introduction}

Skin plays a key role in separation of human body from external environment. This is mainly due to stratum corneum, the outermost layer made of dead cells (corneocytes) surrounded by a lipid intercellular medium. Stratum corneum is organised into a brick-and-mortar-like structure where the bricks are corneocytes and the mortar is made of the intercellular lipids. Such highly hydrophobic structure protects body from excessive water loss on one hand and from pathogen and xenobiotic absorption on the other. Because of this hydrophobic barrier, skin absorption of simple inorganic ions has long been believed limited, or even impossible [1]. However, several recent studies have proved this concept wrong [2,3]. Recent work by Paweloszek et al. [4] shed new light on the possible mechanisms of halide ion transport across the skin including facilitated transport in viable skin by ionic channels and ion transporters. Skin absorption of ionic species is much more complex than just passive diffusion as described for various hydrophobic molecules because of the complex skin structure organized in layers. Outermost poorly hydrated stratum corneum with high content of lipids could indeed act as a simple passive barrier. On the other hand, viable epidermis is characterised by higher water content (up to $70 \%$ ) and living cells producing variety of proteins $[5,6]$. Similar water content is found in dermis that includes collagen and elastin in the intercellular matrix. Therefore, various types of skin-ion interactions are possible.

Anions interacting with biological systems have been classified in Hofmeister series $\left(\mathrm{F}^{-}, \mathrm{Cl}^{-}, \mathrm{Br}^{-}\right.$, $\mathrm{NO}_{3}{ }^{-}, \mathrm{I}^{-}, \mathrm{SCN}^{-}, \mathrm{ClO}_{4}{ }^{-}$) based on their interactions with macromolecules in aqueous solutions [7]. Ions binding to organic materials is related to their "salting in" or "salting out" properties [8]. The Hofmeister series classifies ions that are strongly hydrated in aqueous solution as "kosmotropic" or "water-structure makers". The solubility of neutral organic molecules is reduced in the presence of kosmotropes (saltingout effect). On the other hand, "chaotropic" ions are poorly hydrated in aqueous solution ("waterstructure breakers"). They interact strongly with non-polar substances, which results in their increased solubility (salting-in effect) [7,9-12]. The properties described by Hofmeister series determine various physicochemical phenomena occurring in biological systems. For instance, binding of anions to model phospholipid bilayers was found to increase following the Hofmeister series of anions and binding of chaotropic ions was reported to affect the lipid packing within bilayers [13,14]. Similar effects can be expected within stratum corneum as it has been disclosed by Paweloszek et al. [15]. Moreover, a significant contribution of facilitated transport of tested ions in viable skin was observed [4]. Beyond these observations, the involved physicochemical phenomena still remain poorly understood issues. It is worth noticing that such different phenomena as anion adsorption/absorption to hydrophobic stratum corneum and facilitated anion transport by proteins depend on the nature of anions in the same order: The Hofmeister series.

The present work addresses mixed solutions of anions where non-ideal mixing (interactions with ions of different chemical nature) influence skin absorption. Some clues regarding salts effects have been given in studies of skin absorption of organic molecules from ionic solutions. Thus, MichaelBaruch et al. [16] reported that skin permeation of neostigmine was enhanced in a concentrationdependent manner in the presence of $\mathrm{LiCl}$ and $\mathrm{NaCl}$. Soon after, Ko et al. [17] studied transdermal penetration of salicylic acid from mixed water-ethanol medium at $\mathrm{pH} 2$ containing sodium salts of inorganic anions classified in lyotropic Hofmeister. They reported increased skin permeation in the presence of $\mathrm{NaBr}, \mathrm{NaI}$ and $\mathrm{NaSCN}$ while other salts had no or a reverse effect. As a whole, the Hofmeister series was not followed; the authors ascribed the effects to interactions in solution [16].

Absorption of halide ions from concentrated aqueous solutions of single or mixed sodium halides ( $\mathrm{NaF}, \mathrm{NaBr}, \mathrm{NaI}$ ) into freshly excised skin was investigated. It has been done on excised porcine skin while keeping its metabolism in a suitable survival medium [18]. Young swine skin is the most accurate 
animal model for human skin in terms of permeation properties [19]. It is recommended for in vitro experiments in diffusion Franz cells. Chloride was excluded from the study because its high endogenous concentration in skin $[4,20]$ causes analysis issues. Indeed, chloride is the most abundant anion in the body, especially in extracellular medium [21]. Cross-effects of halides on one another's on skin absorption were investigated in binary and ternary mixtures. Experimental results were exploited in a $\{3,2\}$ simplex-lattice design model allowing quantitative statistical analysis.

\section{Materials and Methods}

\subsection{Chemicals}

$\mathrm{NaF}, \mathrm{NaBr}$ and $\mathrm{NaI}$ were purchased from Fisher Scientific (Illkirch, France). Aqueous solutions of salts were prepared in ultrapure water (resistivity $>18 \mathrm{M} \Omega \cdot \mathrm{cm}$ at $25^{\circ} \mathrm{C}$ ) by weighing, providing concentrations expressed as molality $\left(\mathrm{mol} \cdot \mathrm{kg}^{-1}\right)$.

\subsection{Water activity and density measurements}

Freezing point depression was measured with a Gonotec Osmomat 3000 freezing point osmometer in order to assess water activity $a_{\mathrm{w}}$. The osmometer was calibrated with pure water. Measurements were performed in triplicate with $50 \mu \mathrm{L}$ of ionic solutions. The reading expressed in Osmol $\mathrm{kg}^{-1}$ units was converted into water activity as

Osmolality $=-\frac{\ln \left(a_{\mathrm{w}}\right)}{M_{\mathrm{mol}, \mathrm{w}}}$

Eq. 1

where $M_{\mathrm{mol}, \mathrm{w}}$ is the molar mass of water $\left(0.018 \mathrm{~kg} \cdot \mathrm{mol}^{-1}\right)$.

Densities of ionic solutions needed for the conversion of molality $\left(\mathrm{m}, \mathrm{mol} \cdot \mathrm{kg}^{-1}\right)$ into concentration $\left(C, \mathrm{~mol} \cdot \mathrm{L}^{-1}\right)$ were measured in triplicate at $32{ }^{\circ} \mathrm{C}$ with a $50 \mathrm{~cm}^{3}$ pycnometer calibrated with pure water at $32{ }^{\circ} \mathrm{C}\left(0.995 \mathrm{~kg} \cdot \mathrm{dm}^{-3}\right)$.

Experimental values of densities and osmolalities are given in Table S1 of Supporting Information.

\subsection{Skin samples preparation}

Full-thickness porcine flank skin explants were obtained from five young female pigs (30 $\pm 1 \mathrm{~kg})$, sacrificed at the École de Chirurgie, University Claude Bernard Lyon 1 (Lyon, France). Freshly excised tissue samples were immediately $(<1 \mathrm{~h})$ used to evaluate skin absorption of anions. The bristles were cut with an electrical clipper, and the explants were rinsed in water. The subcutaneous adipose tissue was carefully removed using a scalpel. Skin samples prepared to the final thickness of $1.35 \pm 0.02 \mathrm{~mm}$ (Micrometer Mitutoyo) and cut into round sections of $3 \mathrm{~cm}^{2}$. Skin integrity was assessed by measuring the Trans Epidermal Water Loss (TEWL) (Tewameter TM210, Monaderm, Monaco). Samples presenting TEWL values larger than $15 \mathrm{~g} \cdot \mathrm{h}^{-1} \cdot \mathrm{m}^{-2}$ after 1 min measurements were discarded. The average TEWL values of skin samples used for experiments was $9.85 \pm 0.50 \mathrm{~g} \cdot \mathrm{h}^{-1} \cdot \mathrm{m}^{-2}$.

\subsection{Permeation and ion distribution in skin layers after 24 h exposure}

Skin absorption was carried out for $24 \mathrm{~h}$ in static Franz diffusion cells. Skin explants were mounted into two-chamber glass cells [22] (exposure area $=2.54 \mathrm{~cm}^{2}$ ) with stratum corneum facing up. Acceptor compartment was filled with $10 \mathrm{~mL}$ of salt-free survival medium ensuring skin viability over the duration of the experiment. The isotonic survival medium called betaine-glucose (BG) [18] contained glucose $1 \mathrm{~g} \cdot \mathrm{L}^{-1}$, betaine $2.99 \mathrm{~g} \cdot \mathrm{L}^{-1}$, and halide-free phosphate buffer at $\mathrm{pH} 7.4\left(\mathrm{NaHCO}_{3} 0.35 \mathrm{~g} \cdot \mathrm{L}^{-1}\right.$, $\left.\mathrm{Na}_{2} \mathrm{HPO}_{4} 0.048 \mathrm{~g} \cdot \mathrm{L}^{-1}, \mathrm{KH}_{2} \mathrm{PO}_{4} 0.060 \mathrm{~g} \cdot \mathrm{L}^{-1}\right)$. Mounted Franz cells were placed in a water bath at $37{ }^{\circ} \mathrm{C}$ 
under magnetic stirring, providing a temperature of $32{ }^{\circ} \mathrm{C}$ at the skin surface due to heat loss. After $30 \mathrm{~min}$ of stabilization, the acceptor fluid was renewed and $1 \mathrm{~mL}$ of aqueous solution of sodium halides was placed on the skin surface. Aqueous solutions of $\mathrm{NaF}, \mathrm{NaBr}$ and $\mathrm{NaI}$ alone, in binary and ternary mixtures at the total concentration of $500 \mathrm{mmol} \cdot \mathrm{kg}^{-1}$ were studied.

At the end of the experiment, Franz cells were dismantled and skin layers were separated. Stratum corneum (SC) was removed using cyanoacrylate glue (Loctite SuperGlue-3, Henkel) spread on a glass plate, according to the method for cyanoacrylate surface biopsies [23]. Then, the viable epidermis (VE) was separated from the dermis (D) by immersing the sample in water at $60{ }^{\circ} \mathrm{C}$ for $45 \mathrm{~s}$. The hot water used for this separation was pooled with the extraction medium of the dermis. The underlying assumption is that extracted ions by hot water mainly came from the dermis, a skin layer of high water content where ions mobility is high. The extraction of ions from each skin layer was performed by sonication for $30 \mathrm{~min}$ at $60 \mathrm{~Hz}$ followed by extraction with a water/dichloromethane $(1: 1 \mathrm{v} / \mathrm{v})$ mixture for $17 \mathrm{~h}$. Recovered quantities of ions were measured in the donor medium (DM), the acceptor medium (AM) and the different skin layers. The background amounts coming from endogenous ions was measured in a control experiment without donor fluid under the same conditions as the samples. They were subtracted from the absorbed amounts of the samples. Each measurement was performed on 9 replicates with skin pieces excised from 5 different pigs according to the OECD guidelines [24].

\subsection{Analysis of ions concentrations}

Collected samples were analysed using ion chromatography (930 Compact IC Flex, Metrohm, Switzerland) equipped with a chemical suppressor and conductivity detection. Elution solvent was $8 \mathrm{mmol} \cdot \mathrm{L}^{-1}$ sodium carbonate (Fisher Scientific, Illkirch, France) in ultrapure water. Metrosep A Supp $5250 / 4.0$ column with an adequate pre-column at a temperature of $35^{\circ} \mathrm{C}$ was used for all analyses. Calibration curve was linear from 0.06 to $2000 \mu \mathrm{mol} \cdot \mathrm{kg}^{-1}\left(R^{2}=0.999\right)$.

\subsection{Data reduction}

Results were expressed as recovered quantities in $\mu \mathrm{mol} \cdot \mathrm{cm}^{-2}$. The mean value and standard error of the mean (sem) were calculated from the $n=9$ repetitions of each sample. $Q_{\mathrm{abs}}$ corresponds to the cumulated absorbed amount of ions recovered in $\mathrm{SC}+\mathrm{VE}+\mathrm{D}+\mathrm{AM}$. Comparison of formulations for their significant differences was performed with a Student $t$-test $(p<0.05)$.

\section{Statistical model for ion mixtures}

A mixture design approach was used to investigate the influence of the ionic composition on the skin permeation of each ion. Such approach allows regular screening of the entire experimental domain while limiting the number of experiments.

The experimental domain corresponds to the full ternary diagram drawn in mole fractions of the three ions ranging from zero to unity. This simplex experimental domain allows accommodating a polynomial equation that describes the studied phenomenon over all compositions of mixed ions $[25,26]$. The used $\{3,2\}$ simplex-lattice consisted of the three vertices and the three binary blends located at the midpoints of the edges. According to the Scheffé's design, six aqueous solutions were tested: three single-anion solutions $\mathrm{NaF}, \mathrm{NaBr}$ and $\mathrm{NaI}$ at the concentration of $500 \mathrm{mmol} \cdot \mathrm{kg}^{-1}$ each, $\left(x_{\mathrm{F}}, x_{\mathrm{Br}}, X_{\mathrm{I}}\right)=(1,0,0),(0,1,0)$ and $(0,0,1)$, and three binary mixtures made of equal molar amounts of both ions, $\left(x_{\mathrm{F}}, x_{\mathrm{Br}}, X_{\mathrm{I}}\right)=(1 / 2,1 / 2,0)$, $(1 / 2,0,1 / 2)$ and $(0,1 / 2,1 / 2)$.

The $\{3,2\}$ simplex-lattice allows estimating the six coefficients of a $\{3,2\}$ polynomial equation; i.e. a second-degree model for a three-component system is written as follows: 
where $\hat{y}$ is the predicted response for each observed anion, $x_{\mathrm{i}}$ are the mole fractions of components $i$ in the mixture $\left(x_{\mathrm{F}}+x_{\mathrm{Br}}+x_{\mathrm{I}}=1\right)$ and the parameters $b_{\mathrm{i}} x_{\mathrm{i}}$ and $b_{\mathrm{ij}} x_{\mathrm{i}} x_{\mathrm{j}}$ are respectively the linear and nonlinear mixing terms. In a first analysis, responses $\hat{y}$ are measured absorbed amounts per unit skin area ( $Q_{\mathrm{abs}}$ for $\mathrm{F}^{-}, \mathrm{Br}^{-}$and $\left.\mathrm{I}^{-}\right)$. Thereafter, a second analysis was performed where the ratio of $Q_{\mathrm{abs}}$ to ion activity was introduced as a new response.

An additional experiment corresponding to the centroid of the simplex (ternary mixture of $\mathrm{NaF}, \mathrm{NaBr}$ and $\mathrm{NaI}$ at $167 \mathrm{mmol} \cdot \mathrm{kg}^{-1}$ each, $\left(x_{\mathrm{F}}, x_{\mathrm{Br}}, X_{\mathrm{I}}\right)=(1 / 3,1 / 3,1 / 3)$, has been added as a check point to test the adequacy of the model.

Multiple linear regression calculations, analysis of variance (ANOVA) and statistical analyses were performed with Umetrics MODDE 12.0 software (Umetrics, Umeå, Sweden).

\section{Results and discussion}

\subsection{Experimental results}

Skin penetration of ions has long been believed limited and most of available research on the subject have considered cations far more often than anions. Previous study by Paweloszek et al. [15] initiated research on cutaneous absorption of halide anions by investigating skin absorption of $\mathrm{NaF}, \mathrm{NaCl}, \mathrm{NaBr}$ and $\mathrm{NaI}$ from aqueous solutions of equal mass concentrations. In the present study, molal concentrations were set equal because they are more closely related to thermodynamic parameters such as activity and chemical potential. Chloride was excluded from experiments for the sake of feasibility and reproducibility. Indeed, chloride anions are abundant endogenous ions in the skin tissue; this causes analysis problems for distinguishing endo- and exogenous ions $[4,20]$.

\subsection{Effect of ionic concentration}

The effect of ionic concentration was firstly addressed. Indeed, a higher concentration in the donor side creates a larger concentration gradient through the skin towards the acceptor medium. The amount of ions penetrating skin was very low compared to the amount in the donor solution, so that ion absorption did not significantly deplete the donor fluid; experiments were performed under "infinite dose" conditions. The concentration in the acceptor medium also increased very little compared to that of the donor. As a consequence, the concentration gradient driving passive diffusion was kept constant over the whole experiment duration. Changing the concentration in the donor fluid changes the concentration gradient to the same extent, so that the flux of passive diffusion might be proportional to the concentration in the donor fluid. The sample concentration scale was molality (number of moles of solute per unit mass of solvent, mol $\cdot \mathrm{kg}^{-1}$ ) throughout the whole paper. As shown in Fig. 1, the total absorbed amount of iodide ions penetrating skin actually did not vary linearly with respect to the concentration. It curved downwards at the highest concentrations. Since the ionic concentrations were fairly high, the activity should be better considered than the concentration. Following Stokes and Robinson [27], the activity coefficient of anions, $\gamma$, includes an electrostatic contribution given by the Debye-Hückel relationship and a contribution coming from ion hydration expressed with the help of the water activity, $a_{\mathrm{w}}$, in compliance with Gibbs-Duhem. For a $1: 1$ electrolyte at $32{ }^{\circ} \mathrm{C}$ and concentrations expressed on the molality scale, it reads [28]

$$
\ln (\gamma)=-\frac{z^{2} e^{3} N_{A v}{ }^{1 / 2}}{4 \pi\left(\varepsilon_{0} \varepsilon k T\right)^{3 / 2}} \frac{\sqrt{I}}{1+b \sqrt{I}}-\frac{n}{2} \ln \left(a_{\mathrm{w}}\right)-\ln \left(1-M_{\mathrm{mol}, \mathrm{w}}(n-2) m\right)
$$


where symbols have their usual meaning. $I=\frac{1000}{2} \sum_{\boldsymbol{i o n s}} \mathbf{z}_{\boldsymbol{i}}{ }^{2} \boldsymbol{C}_{\boldsymbol{i}}$ is the ionic strength; $b$ is a constant taken as $1.2 \mathrm{~L}^{1 / 2} \cdot \mathrm{mol}^{-1 / 2}[28] ; M_{\mathrm{mol}, \mathrm{w}}=0.018 \mathrm{~kg} \cdot \mathrm{mol}^{-1}$ is the molar mass of water; and $m$ is the molality of solutions. There is a large variability of experimental values of hydration numbers. The hydration number of sodium halides, $n$, was taken from mean values compiled by Israelachvili [29]: $n=7$ for NaF, $n=6$ for $\mathrm{NaBr}$ and $n=5$ for NaI. Hydration numbers are close to each other because the main contribution comes from the hydration of the sodium cation. The mean value of $n=6$ was taken for the mixtures of all sodium halides. It is noticed that the Debye-Hückel part of Eq. 3 is expressed as a function of molarity $\left(C_{\mathrm{i}}, \mathrm{mol} \cdot \mathrm{dm}^{-3}\right)$ whereas the two other terms are functions of the molality $(\mathrm{m}$, $\left.\mathrm{mol} \cdot \mathrm{kg}^{-1}\right)$. Conversion from one into another is made using measured densities.

The calculation of the activity of mixed ions is a difficult task because too many parameters have to be taken into account. A clear review of the topic is given by Blandamer et al. [30]. The case is especially dramatic when mixed ions have different valences. In the present case, only monovalent anions having related properties (halides) are mixed; the cationic counter-ion is sodium in every case. The activity coefficients were calculated considering mixed ions as single species.

The relationship between absorbed amount and ion activity in the donor medium was linear (Fig. 1). Since the activity correction only considers interactions taking place in bulk aqueous solution, any supplementary non-ideal behaviour of ions absorption from mixed ionic solutions should come from specific interactions operating inside skin.

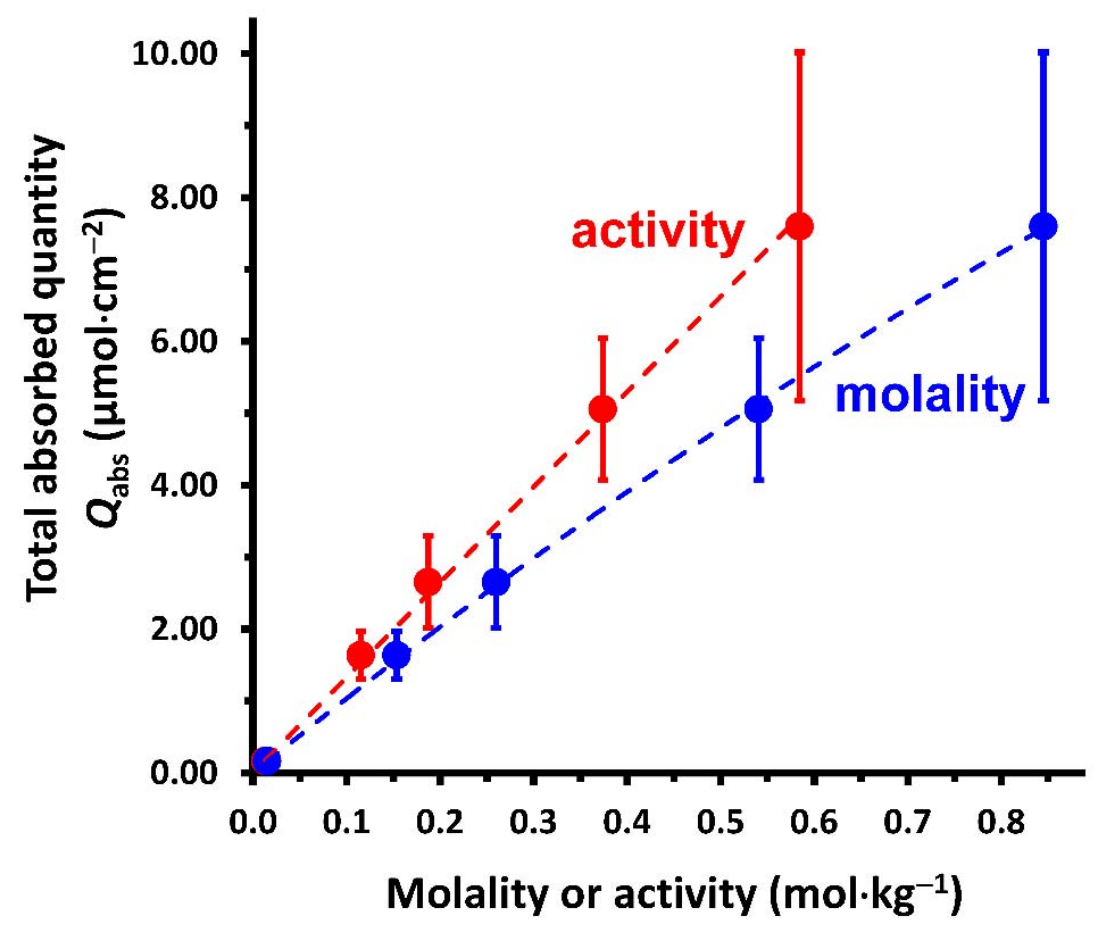

Fig. 1. Total absorbed amount of iodide ions $\left(\mu \mathrm{mol} \cdot \mathrm{cm}^{-2}\right)$ after $24 \mathrm{~h}$ exposure to sodium iodide solutions as a function of iodide concentration (blue) and iodide activity (red).

As a consequence of the linear behaviour with respect to ions activity, it makes sense to consider the ratio of the absorbed amount to ion activity when experiments at different ionic concentrations are compared. Similar ratio has been introduced a long time ago as the permeation coefficient $k_{\mathrm{p}}$ defined as the ratio of the permeation flux in steady state regime to the concentration in the donor medium [31]. We define here the "absorption coefficient", $k_{\mathrm{abs}}$, as the ratio of the total absorbed amount after $24 \mathrm{~h}$ exposure to the activity in the donor solution as: 


\subsection{Absorption of single and mixed anions}

The total absorbed quantity of halide anions tested alone at $500 \mathrm{mmol} \cdot \mathrm{kg}^{-1}$ did not differ significantly (Fig. 2). The same was observed for concentrations of $250 \mathrm{mmol} \cdot \mathrm{kg}^{-1}$ (data not shown). The present results differ from the ones reported previously because the studied solutions were containing the same mass concentration (ppm) for each anion [15] instead of the present molality concentration scale. Given the large differences in molar masses of $\mathrm{F}\left(19 \mathrm{~g} \cdot \mathrm{mol}^{-1}\right), \mathrm{Br}\left(80 \mathrm{~g} \cdot \mathrm{mol}^{-1}\right)$ and $\mathrm{I}\left(127 \mathrm{~g} \cdot \mathrm{mol}^{-1}\right)$, it is easy to notice that conversion into molar concentrations makes the observed variations weaker. Moreover, the survival medium used as the acceptor medium was also different: ultrapure water supplemented with glucose only created a flux of ions towards the hypotonic acceptor solution [15]. In the present study, the acceptor medium was an isotonic composition ensuring equilibrium of osmotic pressure with skin cells and longer viability of skin samples [18].

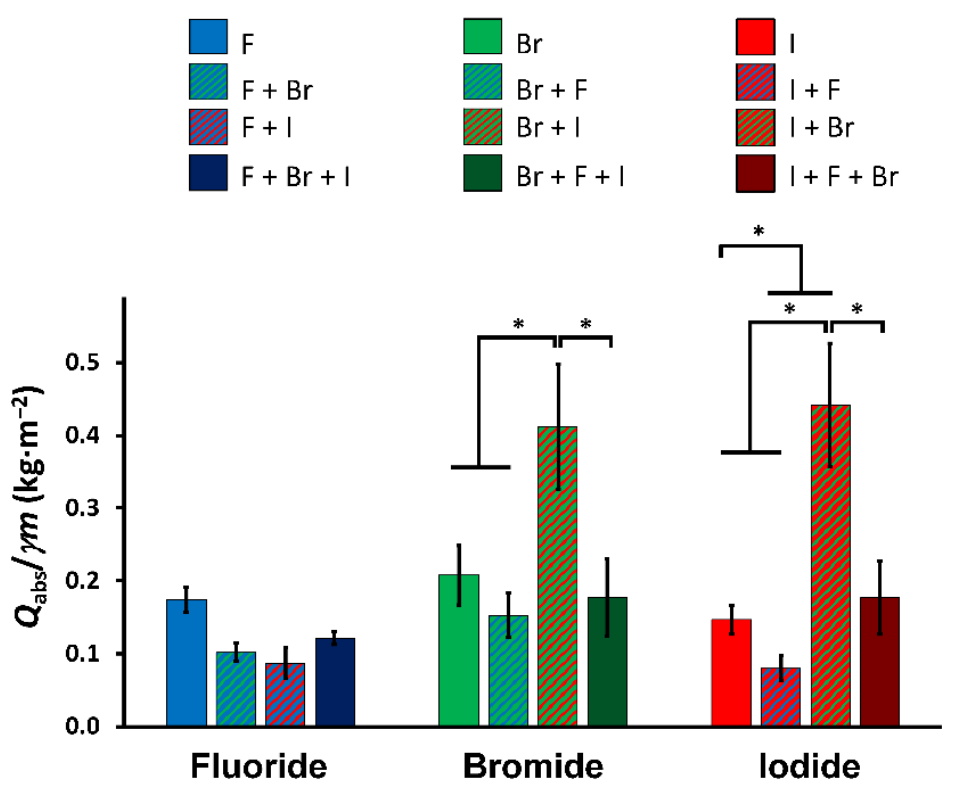

Fig. 2. Absorption coefficient $k_{\mathrm{abs}}=Q_{\mathrm{abs}} / 2 \mathrm{~m}\left(\mathrm{~kg} \cdot \mathrm{m}^{-2}\right)$ after $24 \mathrm{~h}$ exposure time for skin exposed to binary and ternary mixtures of halide salts compared to single halide salt as control. Data represent mean \pm sem carried out on $n=9$ individual skin explants taken from 5 different pigs. Asterisks $\left(^{*}\right)$ indicate $p<0.05$ in $t$-test analysis.

Investigation of skin permeability for anions in binary mixtures revealed significant differences in their absorption depending on the composition. Synergistic effects were observed for mixtures of $\mathrm{NaBr}$ and $\mathrm{NaI}$. The values of $k_{\mathrm{abs}}$ of both anions were significantly higher in the case of paired salts than alone. These observations agree with Ko et al. [17] who studied the effects of addition of $\mathrm{NaF}, \mathrm{NaCl}, \mathrm{NaBr}$, $\mathrm{NaI}$ on skin penetration of salicylic acid. They found that addition of $\mathrm{NaBr}$ or $\mathrm{NaI}$ to the vaseline-based formulation significantly increased absorption of an organic compound through murine skin in vitro and rabbit skin in vivo. On the contrary, addition of $\mathrm{NaF}$ and $\mathrm{NaCl}$ decreased it. Similarly, the present $k_{\mathrm{abs}}$ values for $\mathrm{NaI}$ were significantly reduced when mixed with $\mathrm{NaF}$. Skin penetration of fluoride was also impaired in the presence of $\mathrm{NaBr}$ and $\mathrm{NaI}$.

Ko et al. [17] also observed that salts increased leaching of sterol off excised skin, thereby impairing the initial hydrophobic barrier. This led to infer that enhanced penetration of salicylic acid in presence of $\mathrm{NaBr}$ or $\mathrm{NaI}$ might be due to the reduction of the barrier function of stratum corneum by 
disorganization of the crystalline structure of its lipids, thereby allowing better swelling. According to Elden [32], lyotropic swelling of collagen also occurs in the order: $\mathrm{F}^{-}<\mathrm{Cl}^{-}<\mathrm{Br}^{-}<\mathrm{SCN}^{-}$. The main presumption from these studies is that penetrating chaotropic ions disorganize the skin barrier and make it more permeable to penetrating ionic or nonionic molecules. There is no experiental evidence of such phenomenon, however. The present study goes deeper into the mechanisms by considering the distribution of anions in skin layers: stratum corneum, viable epidermis, dermis, and acceptor medium (SC, VE, D and AM). Indeed, anions distribution in skin layers after $24 \mathrm{~h}$ exposure (Fig. 3) was also altered depending on the composition of tested solution. Simultaneous exposure to $\mathrm{Br}^{-}$and $\mathrm{I}^{-}$led to increased recovery in $\mathrm{AM}$ as compared to $\mathrm{Br}^{-}$and $\mathrm{I}^{-}$alone. This was associated with reduced values in $\mathrm{D}, \mathrm{VE}$ and especially SC, indicating lower tendency for accumulation within the tissues. These observations contradict the postulated idea of a disorganization of skin structure by the presence of absorbed anions. Indeed, structural modification of skin layers would require accumulation of ions inside them. On the other hand, the trends are reversed for iodide distribution in the presence of $\mathrm{NaF}$ : recovery in AM slightly decreased for mixed ions and absorbed amount in SC increased. This effect was less pronounced in the case of $\mathrm{NaF}+\mathrm{NaBr}$.

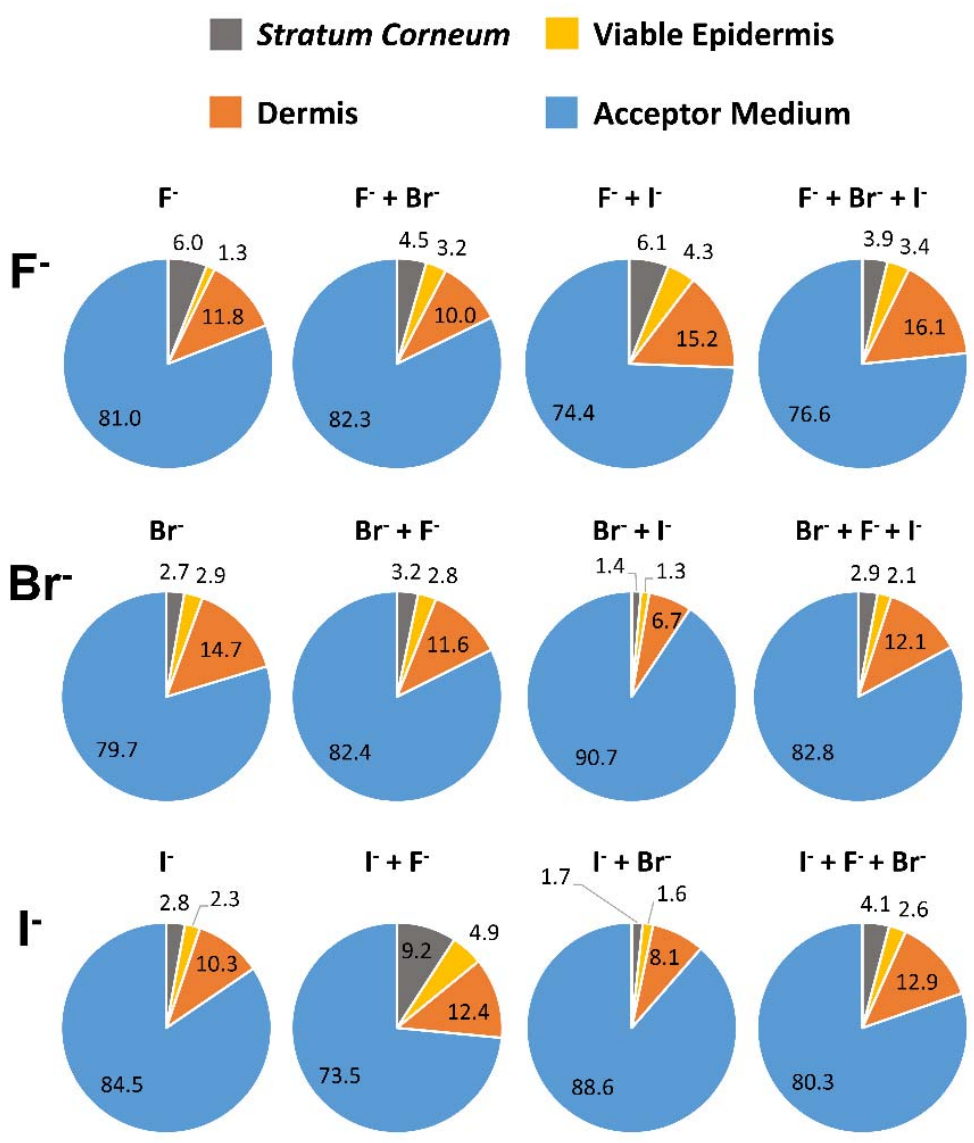

Fig. 3. Distribution of halide anions in skin layers after $24 \mathrm{~h}$ exposure. Data represent mean value of absorbed amount expressed as a percentage of the total absorbed amount $Q_{\text {abs. }}$ Results are means of $n=9$ independent measurements.

The observed effects are quite complex because they result from several contributions: interactions between ions in the bulk donor solution, adsorption of ions to the skin surface, transfer into stratum corneum and passive diffusion inside it, facilitated transport in the viable epidermis with the help of membrane proteins in keratinocytes (ion channels, ion transporters). 
Interactions in bulk solution are considered non-specific and are accounted for by the activity coefficient involving electrostatic and ion hydration contributions. Facilitated transport in viable skin is predominant; indeed, Paweloszek et al. [4] disclosed a large difference between viable and non-viable skin. Passive diffusion through stratum corneum is necessary for ions to reach viable epidermis where facilitated transport operates. Most elementary steps for ion absorption are interdependent. Transport phenomena depend on ion type in the same way according to Hofmeister series. The best strategy for addressing such a complexity is a global approach undertaken here using an experimental design.

\subsection{Statistical Modelling}

An alternative way to exploit experimental results consists in fitting them to statistical models. The beneficial input of such data analysis is a rigorous statistical framework. The outcome is a quantitative parametric assessment of non-ideal behaviour through the coefficients of the Scheffé $\{3,2\}$ polynomial equation (Eq. 2). The $\hat{y}$-responses are either absorbed amounts $Q_{\text {abs }}$ or absorption coefficients $k_{\text {abs }}=$ $Q_{\text {abs }} / 2 m$. Experimental values of $Q_{\text {abs }}$ and $k_{\text {abs }}$ are given in Table S2 of Supporting Information. They must fully fill a matrix containing absorption data of ions thought some of them are not present in the solution of mixed ions. As example, a binary mixture of fluoride and bromide does not contain iodide, but the matrix for iodide absorption data does contain a figure pertaining to iodide absorption from a mixed solution of fluoride and bromide. This is mandatory to fully fill the $\hat{y}$-response matrix in the mathematical framework of Scheffé simplex-lattice design. This is easy to manage when $Q_{\text {abs }}$ is considered as a response: $Q_{\text {abs }}$ is zero when the anion is not present. When responses are absorption coefficients, the correct values are $\lim _{m \rightarrow 0}\left(\frac{Q_{\mathrm{abs}}}{m}\right)$. They were obtained by extrapolation from experiments at several concentrations. Although those concentrations were fairly high $(>100 \mathrm{mM})$ for ensuring enough experimental accuracy, extrapolation to infinite dilution was quite robust, owing to the almost linear dependence of $Q_{\text {abs }}$ with respect to $\gamma m$ in the low concentration range. A first approach was to determine the coefficients of the polynomial using the six experiments of the $\{3,2\}$ simplex-lattice design for the skin absorption of each anion. Since there are mixtures where some ions are absent, their coefficient in the polynomial is undetermined, and it has been removed from the polynomial model. Keeping these coefficients led to statistically non-significant values $(p>0.75)$. As example, because there is no $\mathrm{I}^{-}$in a binary mixture of $\mathrm{F}^{-}$and $\mathrm{Br}^{-}$, the term containing the coefficient $b_{\mathrm{FBr}}$ was removed from the polynomial pertaining to $Q_{\text {abs }}$ or $k_{\text {abs }}$ of iodide. The modified Eq. 2 became the set of Eqs 5 to 7 for $Q_{\text {abs }}$ and Eqs 8 to 10 for $k_{\text {abs: }}$ :

$$
\begin{array}{ll}
Q_{\mathrm{abs}}\left(\mathrm{F}^{-}\right)=a_{\mathrm{F}} X_{\mathrm{F}}+a_{\mathrm{Br}} X_{\mathrm{Br}}+a_{\mathrm{I}} X_{\mathrm{I}}+a_{\mathrm{FBr}} X_{\mathrm{F}} X_{\mathrm{Br}}+a_{\mathrm{FI}} X_{\mathrm{F}} X_{\mathrm{I}} & \text { Eq. } 5 \\
Q_{\mathrm{abs}}\left(\mathrm{Br}^{-}\right)=a_{\mathrm{F}} X_{\mathrm{F}}+a_{\mathrm{Br}} X_{\mathrm{Br}}+a_{\mathrm{I}} X_{\mathrm{I}}+a_{\mathrm{FBr}} X_{\mathrm{F}} X_{\mathrm{Br}}+a_{\mathrm{Br}} X_{\mathrm{Br}} X_{\mathrm{I}} & \text { Eq. } 6 \\
Q_{\mathrm{abs}}\left(\mathrm{I}^{-}\right)=a_{\mathrm{F}} X_{\mathrm{F}}+a_{\mathrm{Br}} X_{\mathrm{Br}}+a_{\mathrm{I}} X_{\mathrm{I}}+a_{\mathrm{FI}} X_{\mathrm{F}} X_{\mathrm{I}}+a_{\mathrm{Br}} X_{\mathrm{Br}} X_{\mathrm{I}} & \text { Eq. } 7 \\
k_{\mathrm{abs}}\left(\mathrm{F}^{-}\right)=b_{\mathrm{F}} X_{\mathrm{F}}+b_{\mathrm{Br}} X_{\mathrm{Br}}+b_{\mathrm{I}} X_{\mathrm{I}}+b_{\mathrm{FBr}} X_{\mathrm{F}} X_{\mathrm{Br}}+b_{\mathrm{FI}} X_{\mathrm{F}} X_{\mathrm{I}} & \text { Eq. } 8 \\
k_{\mathrm{abs}}\left(\mathrm{Br}^{-}\right)=b_{\mathrm{F}} X_{\mathrm{F}}+b_{\mathrm{Br}} X_{\mathrm{Br}}+b_{\mathrm{I}} X_{\mathrm{I}}+b_{\mathrm{FBr}} X_{\mathrm{F}} X_{\mathrm{Br}}+b_{\mathrm{Br}} X_{\mathrm{Br}} X_{\mathrm{I}} & \text { Eq. } 9 \\
k_{\mathrm{abs}}\left(\mathrm{I}^{-}\right)=b_{\mathrm{F}} X_{\mathrm{F}}+b_{\mathrm{Br}} X_{\mathrm{Br}}+b_{\mathrm{I}} X_{\mathrm{I}}+b_{\mathrm{FI}} X_{\mathrm{F}} X_{\mathrm{I}}+b_{\mathrm{Br}} X_{\mathrm{Br}} X_{\mathrm{I}} & \text { Eq. } 10
\end{array}
$$

$Q_{\text {abs }}$ was first analysed because this was the actually measured parameter. The models for $Q_{\text {abs }}$ (Eqs 5, 6 and 7) were first used to predict the amounts of absorbed ions for the centroid experiment (ternary mixture) as a validation tool of the model: residuals of $0.5,-1.6$ and $-1.3 \mu \mathrm{mol} \cdot \mathrm{cm}^{-2}$ were respectively obtained for $Q_{\text {abs }}\left(\mathrm{F}^{-}\right), Q_{\text {abs }}\left(\mathrm{Br}^{-}\right)$and $Q_{\text {abs }}\left(I^{-}\right)$. They were considered satisfactory compared to the experimental standard deviation. The centroid experiment was then used in combination with the 6 experiments of the simplex-lattice design to fit once again the models for $Q_{\text {abs }}$ and $k_{\text {abs }}$ to the full experimental data. The best statistical models (characterized by highest values of $R^{2}$ ) are presented in 
Table 1. For each model, ANOVA analysis indicated the high significance of the fits $(p<0.05)$; and the corresponding determination coefficient $R^{2}$ greater than 0.97 (Table 1) also showed their satisfactory adequacy.

Once validated, the models were used to plot the estimated surface contours of the studied responses in the investigated experimental domain, i.e. in a classical ternary diagram in mole fractions of ions (Fig. 4) where the colour scale stands for $Q_{\text {abs. }}$

The variation of $Q_{\text {abs }}$ of $\mathrm{F}^{-}$in Fig. 4 shows a continuous increase of $Q_{\text {abs }}$ as the mole fraction of fluoride increases, which is the obvious consequence of the increase of fluoride concentration in the donor solution. The iso- $Q_{\text {abs }}$ lines are almost horizontal in the triangular presentation where pure fluoride is at the apex, showing that the effects of bromide and iodide ions are identical and negligible. As a whole, there is no specific effect of mixed anions. Conversely, absorptions of bromide and iodide anions show a maximum in the presence of the other. There is no apparent effect of fluoride. The statistical analysis disclosed a specific interaction between bromide and iodide that caused enhanced skin absorption of these anions. The maxima of absorption occur when the solution is rich in the observed anion because of the trivial effect of the higher concentration of the observed anion as the corner of pure anion is approached. This analysis considering $Q_{\mathrm{abs}}$ includes contributions of the concentration of observed anions and specific interactions with their partners. A quantitative approach is to consider the $a_{\mathrm{ij}}$ coefficients (Table 1) that have statistical significance. The $a_{\mathrm{F}}, a_{\mathrm{Br}}$ and $a_{\mathrm{I}}$ coefficients of the same anions (e.g. $a_{\mathrm{F}}$ for $Q_{\mathrm{abs}}$ of $\mathrm{F}^{-}$) are highly significant $(p \leq 0.01)$ and positive. They reflect the trivial effects of increased absorption as a function of concentration. The $a_{\mathrm{F}}, a_{\mathrm{Br}}$ and $a_{\mathrm{I}}$ coefficients of different anions (e.g. $a_{\mathrm{F}}$ for $Q_{\mathrm{abs}}$ of $\mathrm{Br}^{-}$) are statistically not significant $(S D$ is larger than the coefficient value and $p=$ 0.93). $a_{\mathrm{FBr}}$, and $a_{\mathrm{FI}}$ negative coefficients are also not significant owing the large $S D$ and $p$-value. The striking feature is the large and significant positive values of $a_{\mathrm{Br}}$, showing a specific interaction between bromide and iodide anions.
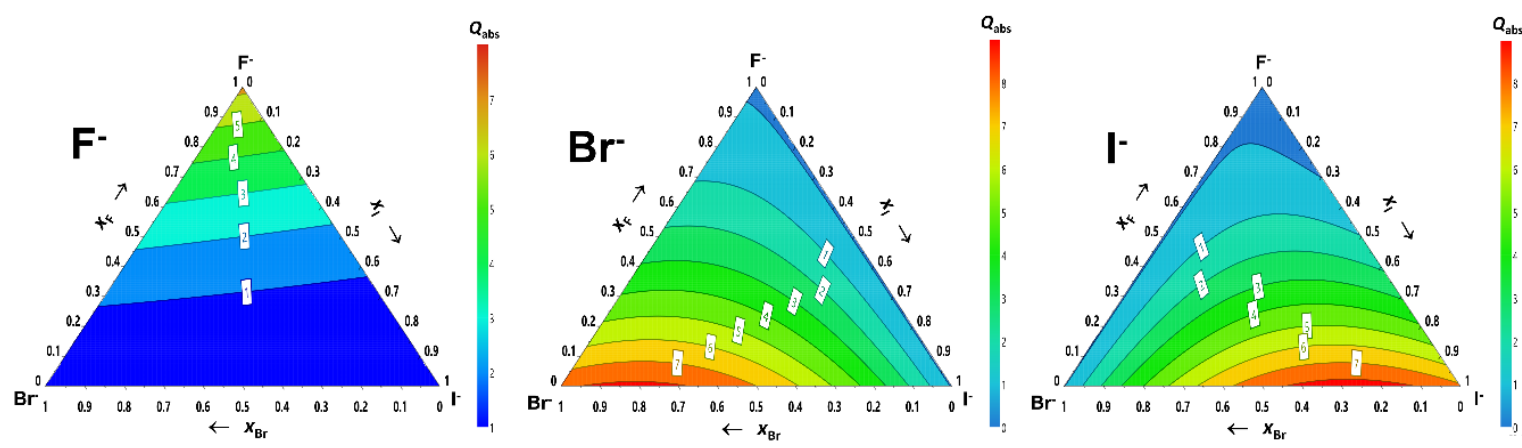

Fig. 4. Contour plots of the predicted absorbed amounts $\left(Q_{\mathrm{abs}}, \mu \mathrm{mol} \cdot \mathrm{cm}^{-2}\right)$ of $\mathrm{F}^{-}, \mathrm{Br}^{-}$and $\mathrm{I}^{-}$ions after 24 h exposure in the composition space of ionic mixtures expressed in mole fractions.

\section{Table 1}

Coefficients $a_{\mathrm{ij}}\left(\mu \mathrm{mol} \cdot \mathrm{cm}^{-2}\right)$ of the $\{3,2\}$ polynomial models of absorbed amounts $Q_{\mathrm{abs}}($ Eqs 5,6 and 7$)$ together with the corresponding determination coefficient $R^{2}$, standard deviation $(S D)$ and $p$-value of the ANOVA test between brackets. All values are given with two digits with no consideration to their statistical significance.

\begin{tabular}{cccc}
\hline Ions $(\mathbf{i}, \mathbf{j})$ & $\mathbf{F}^{-}$ & $\mathbf{B r}^{-}$ & $\mathbf{I}^{-}$ \\
$\mathbf{F}^{-}$ & $\boldsymbol{a}_{\mathrm{ij}}(\boldsymbol{S D}, \boldsymbol{p})$ & $\boldsymbol{a}_{\mathrm{ij}}(\boldsymbol{S D}, \boldsymbol{p})$ & $\boldsymbol{a}_{\mathrm{ij}}(\boldsymbol{S D}, \boldsymbol{p})$ \\
$\mathbf{B r}^{-}$ & $\mathbf{6 . 2 6}(0.3,0.001)$ & $-\mathbf{0 . 0 8}(0.9,0.93)$ & $\mathbf{- 0 . 0 7}(0.7,0.93)$ \\
$\mathbf{I}^{-}$ & $\mathbf{0 . 0 3}(0.3,0.93)$ & $\mathbf{7 . 8 0}(0.9,0.01)$ & $-\mathbf{0 . 0 7}(0.7,0.93)$ \\
$\mathbf{F}^{-} / \mathbf{B r}^{-}$ & $\mathbf{0 . 0 3}(0.3,0.93)$ & $-\mathbf{0 . 0 8}(0.9,0.93)$ & $\mathbf{7 . 2 0}(0.78,0.01)$ \\
$\mathbf{F}^{-} / \mathbf{I}^{-}$ & $-\mathbf{3 . 5 3}(1.4,0.13)$ & $-\mathbf{6 . 5 0}(4.3,0.27)$ & - \\
$\mathbf{B r}^{-} / \mathbf{I}^{-}$ & $-\mathbf{5 . 6 5}(1.4,0.06)$ & - & $-\mathbf{1 0 . 1}(3.6,0.11)$ \\
\hline
\end{tabular}


The global statistical analysis of skin absorption of mixed anions disclosed a specific interaction between them, but neither had it allowed inferring the nature of the interactions, nor the location of them in the complex layered structure of skin (donor solution, stratum corneum, viable epidermis and dermis). A refined analysis was performed considering the absorption coefficient $k_{\mathrm{abs}}=Q_{\mathrm{abs}} / 2 m$ in place of $Q_{\mathrm{abs}}$. This suppresses the effects of the concentration of observed anion and interactions taking place in the bulk donor solution through the activity coefficient. The same statistical analysis as for $Q_{\text {abs }}$ was made. Results are given in Table 2 and Fig. 5.

Substitution of $k_{\mathrm{abs}}$ for $Q_{\mathrm{abs}}$ eliminates the effects of ion concentrations in a similar way as applying a background flattening image processing to the triangular composition maps of Fig. 4. The sole remaining effects are specific cross-interactions. The contour plot for fluoride is almost flat with a constant value of $k_{\mathrm{abs}}=0.1 \mathrm{~kg} \cdot \mathrm{m}^{-2}$ (Fig. 5). This feature shows the overall absence of specific interactions of bromide and iodide with fluoride. Conversely again, the $k_{\text {abs }}$ maps of bromide and iodide anions show a maximum at the centre of the bottom side. Substitution of $k_{\text {abs }}$ for $Q_{\text {abs }}$ shifted the location of the absorption maximum at the bottom side of the triangle from close to the vertex of adsorbed ion to the mid-point of the $\mathrm{Br}-\mathrm{I}$ axis, $\left(x_{\mathrm{F}}, x_{\mathrm{Br}}, X_{\mathrm{I}}\right)=(0,1 / 2,1 / 2)$. Such symmetrical position suggests a $1: 1$ stoichiometry of specific interaction between bromide and iodide anions that could not be detected in the raw $Q_{\mathrm{abs}}$ map. Many of the $b_{\mathrm{ij}}$ coefficients (Table 2) have poor statistical significance. So, the significance of outcoming $b_{\mathrm{ij}}$ coefficients is first discussed. The distribution of $p$-values was definitely bimodal with either very large or moderate values. $p<0.1$ was taken for claiming significance in the present case instead of the classical criterion $p<0.05$ for the full population of low/moderate $p$-values is taken as significant. Under this condition, the $b_{\mathrm{F}}, b_{\mathrm{Br}}$ and $b_{\mathrm{I}}$ coefficients are significant $(p<0.07)$. The $b_{\mathrm{BrI}}$ and $b_{\mathrm{IBr}}$ are also of medium significance; they assume a high positive value. The negative $b_{\mathrm{FBr}}$ and $b_{\mathrm{FI}}$ coefficients involving the $\mathrm{F}^{-}$anion are not significant. They nevertheless give a contribution to other coefficients in the statistical model. Thus, it is clear from the flat $\mathrm{F}^{-}$map (Fig. 5) that skin absorption of fluoride was not influenced by the presence of other ions, so that the positive values of $b_{\mathrm{F}}$ coefficients contradicted the overall features of the $\mathrm{F}^{-}$map of $k_{\mathrm{abs}}$. It is suspected that the effects of the positive values of $b_{\mathrm{F}}$ and the negative values of $b_{\mathrm{FBr}}$ and $b_{\mathrm{FI}}$ compensated such that the balance is null. Because of the lack of statistical significance of $b_{\mathrm{FBr}}$ and $b_{\mathrm{FI}}$ coefficients for absorption of all anions, it is considered that the positive values of $b_{\mathrm{F}}$ are biases coming from a too large variability of skin absorption measurements. Such high variability is intrinsic of experiments performed on skin and living matter in general.
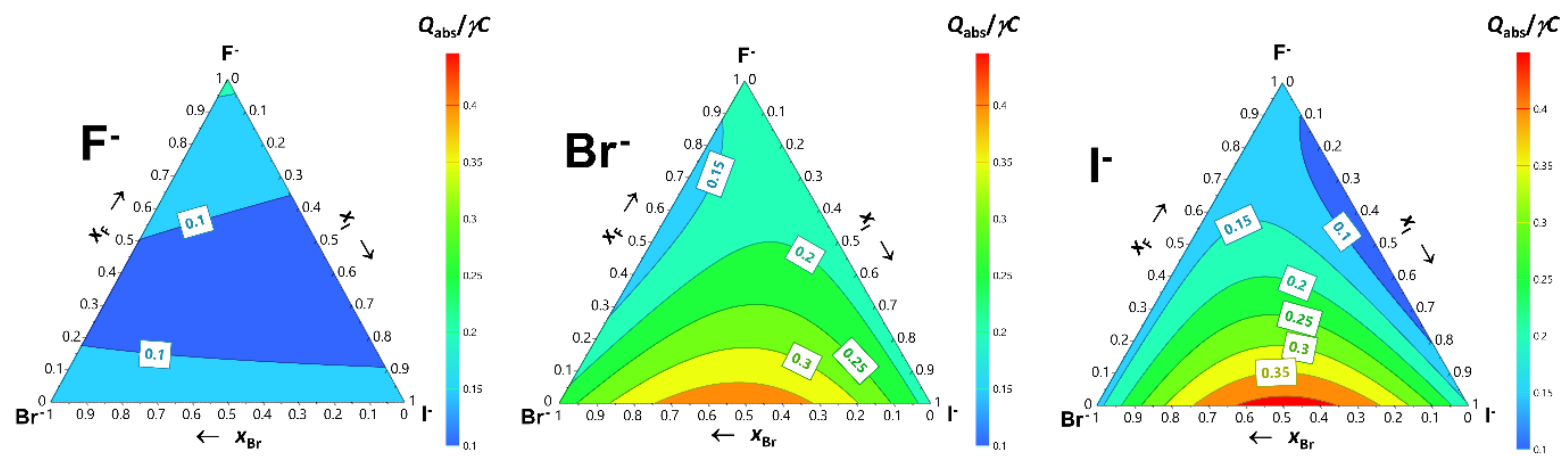

Fig. 5. Contour plots of the predicted absorption coefficients $\left(k_{\mathrm{abs}}=Q_{\mathrm{abs}} / \gamma C, \mathrm{~kg} \cdot \mathrm{m}^{-2}\right)$ of $\mathrm{F}^{-}, \mathrm{Br}^{-}$and $\mathrm{I}^{-}$ions after $24 \mathrm{~h}$ exposure in the composition space of ionic mixtures expressed in mole fractions.

Table 2 
Coefficients $b_{\mathrm{ij}}\left(\mathrm{kg} \cdot \mathrm{m}^{-2}\right)$ of the $\{3,2\}$ polynomial models of absorption coefficients $k_{\mathrm{abs}}$ (Eqs 8, 9 and 10) together with the corresponding determination coefficient $R^{2}$, standard deviation $(S D)$ and $p$-value of the ANOVA test between brackets. All values are given with two digits with no consideration to their statistical significance.

\begin{tabular}{cccc}
\hline Ions $(\mathbf{i}, \mathbf{j})$ & $\begin{array}{c}\mathbf{F}^{-} \\
\boldsymbol{b}_{\mathrm{ij}}(\boldsymbol{S} \boldsymbol{D}, \boldsymbol{p})\end{array}$ & $\begin{array}{c}\mathbf{B r}^{-} \\
\boldsymbol{b}_{\mathrm{ij}}(\boldsymbol{S} \boldsymbol{D}, \boldsymbol{p})\end{array}$ & $\begin{array}{c}\mathbf{I}^{-} \\
\boldsymbol{b}_{\mathrm{ij}}(\boldsymbol{S} \boldsymbol{D}, \boldsymbol{p})\end{array}$ \\
\hline $\mathbf{F}^{-}$ & $\mathbf{0 . 1 7}(0.017,0.010)$ & $\mathbf{0 . 1 7}(0.048,0.071)$ & $\mathbf{0 . 1 7}(0.041,0.051)$ \\
$\mathbf{B r}^{-}$ & $\mathbf{0 . 1 2}(0.016,0.016)$ & $\mathbf{0 . 2 2}(0.053,0.056)$ & $\mathbf{0 . 1 7}(0.041,0.051)$ \\
$\mathbf{I}^{-}$ & $\mathbf{0 . 1 2}(0.016,0.016)$ & $\mathbf{0 . 1 7}(0.048,0.071)$ & $\mathbf{0 . 1 8}(0.044,0.055)$ \\
$\mathbf{F}^{-} / \mathbf{B r}^{-}$ & $-\mathbf{0 . 1 4}(0.080,0.21)$ & $-\mathbf{0 . 2 8}(0.24,0.37)$ & - \\
$\mathbf{F}^{-} / \mathbf{I}^{-}$ & $-\mathbf{0 . 2 0}(0.080,0.13)$ & - & $-\mathbf{0 . 4 8}(0.20,0.14)$ \\
$\mathbf{B r}^{-} / \mathbf{I}^{-}$ & - & $\mathbf{0 . 7 6}(0.24,0.089)$ & $\mathbf{0 . 9 6}(0.20,0.042)$ \\
\hline $\boldsymbol{R}^{\mathbf{2}}$ & $\mathbf{0 . 8 6}$ & $\mathbf{0 . 8 8}$ & $\mathbf{0 . 9 5}$
\end{tabular}

Refinement of the analysis by considering $k_{\text {abs }}$ may allow discrimination between the ion effects coming from alteration of the medium (ionic strength, dielectric properties...) through the $b_{\mathrm{F}}, b_{\mathrm{Br}}$ and $b_{\mathrm{I}}$ coefficients, and from specific interactions through the $b_{\mathrm{FBr}}, b_{\mathrm{FI}}$ and $b_{\mathrm{BrI}}$ coefficients. This was successful and led to supplementary conclusions:

- Owing to the linear dependence of $Q_{\text {abs }}$ with respect to activity for $\mathrm{F}^{-}, \mathrm{Br}^{-}$and $\mathrm{I}^{-}$(Fig. 1), the values of $b_{\mathrm{F}}, b_{\mathrm{Br}}$ and $b_{\mathrm{I}}$ should be null. The positive values of $b_{\mathrm{F}}, b_{\mathrm{Br}}$ and $b_{\mathrm{I}}\left(\approx 0.2 \mathrm{~kg} \cdot \mathrm{m}^{-2}\right.$ for all anions $)$ cannot be considered as a non-specific effect of the presence of other ions inside skin. Their contribution is compensated by negative $b_{\mathrm{ij}}$ coefficients that are not significant as it has been shown from the flat $k_{\mathrm{abs}}$ map for $\mathrm{F}^{-}$absorption.

- The high positive values of $b_{\mathrm{Br}}$ indicates a strong specific interaction between bromide and iodide. This is the main outcome of the statistical analysis.

A specific interaction operating between bromide and iodide was already inferred from the raw experimental data. The statistical analysis showed that this was the sole significant specific interaction. The absorption coefficient $k_{\mathrm{abs}}$ of $\mathrm{Br}^{-}$in the presence of an equal amount of $\mathrm{I}^{-}$was $0.41 \mathrm{~kg} \cdot \mathrm{m}^{-2}$ against $0.21 \mathrm{~kg} \cdot \mathrm{m}^{-2}$ for $\mathrm{Br}^{-}$alone (Table S2 and Fig 2), which was twice an absorption enhancement caused by non-ideal mixing. In the same symmetrical case, $k_{\mathrm{abs}}$ of $\mathrm{I}^{-}$increased by a factor of three in the presence of an equal amount of $\mathrm{Br}^{-}\left(k_{\mathrm{abs}}=0.44 \mathrm{~kg} \cdot \mathrm{m}^{-2}\right.$ against $0.146 \mathrm{~kg} \cdot \mathrm{m}^{-2}$ for $\mathrm{I}^{-}$alone). Such interactions between bromide and iodide caused an absorption enhancement of their partner by a factor of 2-3 with respect to the case of ideal mixing.

\subsection{Discussion}

The location of disclosed interactions is difficult to discuss in details despite the distribution of ions stored in the different skin layers has been measured (Fig. 3). As main the feature, very few figures show significant differences. The main contributions to differences of total absorbed amount $Q_{\mathrm{abs}}$ come from the amounts that reached the acceptor medium. The very small differences of amounts in SC suggest that $\mathrm{SC}$ is just a passive diffusion barrier where permeation is only driven by the concentration gradient. There is no accumulation of ions inside SC, showing that there is no specific binding of anions there. There are also small differences of ion amounts in viable epidermis and dermis. It is known that these skin layers are not passive diffusion barriers because they contain ion transporters contributing a lot to the overall absorption [15]. The absorbed amounts per unit skin area can be converted into concentrations in $\mathrm{mmol} \cdot \mathrm{L}^{-1}$ taking the thickness of SC, VE and D as $20 \mu \mathrm{m}, 50 \mu \mathrm{m}$ and $1.25 \mathrm{~mm}$. The result is a smooth concentration gradient along skin depth in all cases (Fig. 6). One consequence is that concentrations in different layers are interdependent. There is no indication of accumulation coming either from specific interactions with skin materials or any ion-specific barrier in SC or VE. 


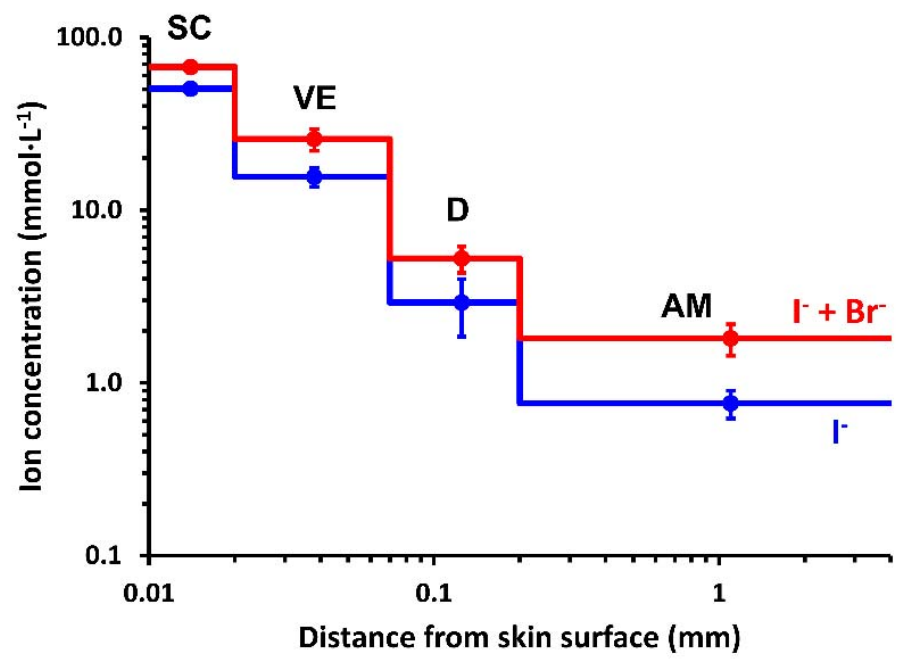

Fig. 6. Concentration profile of iodide ions inside skin layers $\left(\mathrm{mmol} \cdot \mathrm{L}^{-1}\right)$ as a function of depth showing the concentration gradient from the surface to the acceptor medium. Concentrations are taken homogeneous in each skin layer, Stratum corneum (SC), Viable epidermis (VE), Dermis (D) and Acceptor medium (AM).

The nature of interactions operating in skin absorption experiments cannot be inferred from the present data analysis that remains a global approach. Non-specific electrostatic interactions do operate in the concentrated donor solution; they have been accounted for by considering the activity coefficients. Ions absorption cannot contribute to electrostatic effects inside skin because the concentrations of penetrating ions inside skin layers were low with respect to the concentrations of endogenous ions [4]. As example taking the thickness of viable epidermis as $50 \mu \mathrm{m}$ and that of dermis as $1.3 \mathrm{~mm}$, the mean concentration of endogenous chloride in viable epidermis and dermis are respectively $200 \mathrm{mmol} \cdot \mathrm{L}^{-1}$ and $15 \mathrm{mmol} \cdot \mathrm{L}^{-1}$; the concentrations of sodium are three times larger and those of potassium are also larger than those of chloride [4]. All presently measured anions concentrations were less than $50 \mathrm{mmol} \cdot \mathrm{L}^{-1}$ in viable epidermis and less than $10 \mathrm{mmol} \cdot \mathrm{L}^{-1}$ in dermis.

A specific interaction between bromide and iodide ions should be considered. These two anions are chaotropic in the Hofmeister series of halides because of their large size and high polarizability allowing strong attractive dispersion interactions and their poor hydration. Hydration of ions [33,34] and dispersion interactions [35] are the two main rationalizing factors for the Hofmeister series. As outlined early by Fajans [36], both phenomena are interrelated as they originate from the size and polarizability of ions [37-39]. Both Hofmeister and anti-Hofmeister behaviours have been reported [40,41]; their concomitance may lead to compensation of effects and even reversal of the global balance. Owing to the poor current knowledge on skin ultrastructure and interactions operating inside skin layers, going farther would require a full account of many interactions before a simplified view retaining only the predominant interactions could be reached. A recent study of water sorption by wood is a successful example of such an approach [42]. Electrostatic repulsions act against association of $\mathrm{Br}^{-}$and $\mathrm{I}^{-}$anions, so that a cation is necessarily involved in the association. Possible complex species may result from the association of $\mathrm{Br}^{-}, \mathrm{I}^{-}$and $\mathrm{Na}^{+}$ions as the $\mathrm{NaBrI}^{-}$monovalent anion. Another option is the association with endogenous magnesium or calcium cations by formation of the neutral $\mathrm{MgBrI}$ and $\mathrm{CaBrI}$ species. Indeed, magnesium and calcium are abundant endogenous ions inside skin, with the presence of a $\mathrm{Ca}^{2+}$ gradient from the stratum basale to the stratum granulosum where it peaks before declining in stratum corneum [43]. On the basis of size and polarizability of ionic partners, $\mathrm{Mg}^{2+}, \mathrm{Ca}^{2+}, \mathrm{Br}^{-}$and $\mathrm{I}^{-}$[37-39], such complex association with magnesium or calcium looks quite a likely mechanism for anions to pass through the stratum corneum. 


\section{Conclusion}

Skin absorption of mixed halide ions shows synergistic effects between chaotropic bromide and iodide anions. This has been clearly disclosed by a statistical analysis of absorption experiments on excised pig skin mounted in diffusion Franz cells. Statistical analysis allowed identification of interacting anions pairs from skin absorption experiments that show a large variability. Considering the ratio of the absorbed amounts to the ion activity discards the trivial contributions of ion concentration and non-specific effects of electrostatic interactions in the aqueous solution; such data reduction process highlights the effects of specific interactions.

It has been inferred that polarizable hydrophobic anions associate in the stratum corneum as complex species together with cations, in particular with magnesium and calcium, in order to counterbalance their electrostatic repulsions.

The present results together with our previous work [15], definitely show that ionic species do penetrate skin. They are of relevance to practical ionic solutions such as spring waters used in cosmetic products.

\section{Declaration of competing interest}

The authors declare that they have no known competing financial interests or personal relationships that could have appeared to influence the work reported in this paper.

\section{Acknowledgements}

MT acknowledges a PhD grant from the University of Lyon.

\section{Appendix A. Supplementary data}

Supplementary material related to this article can be found in the on line version of Supporting Information file

\section{References}

[1] J. Hadgraft and C. Valenta, Int. J. Pharm., 200 (2000) 243-247.

[2] T. D. La Count and G. B. Kasting, J. Pharm. Sci., 102 (2013) 2241-2253.

[3] M.-A. Bolzinger, C. Bolot, G. Galy, A. Chabanel, J. Pelletier and S. Briançon, Int. J. Pharm., 402 (2010) 44-49.

[4] R. Paweloszek, S. Briançon, Y. Chevalier, N. Gilon-Delepine, J. Pelletier and M.-A. Bolzinger, Pharm. Res., 33 (2016) 1564-1575.

[5] T. von Zglinicki, M. Lindberg, G. M. Roomans and B. Forslind, Acta Derm. Venerol., 116 (1993) 434-442.

[6] R. R. Warner, M. C. Myers and D. A. Taylor, J. Invest. Dermatol., 90 (1988) 218-224.

[7] M. G. Cacace, E. M. Landau and J. J. Ramsden, Q. Rev. Biophys., 3 (1997) 241-277.

[8] W. Kunz, P. Lo Nostro and B. W. Ninham, Curr. Opin. Colloid Interface Sci., 9 (2004) 1-18.

[9] K. D. Collins and M. W. Washabaugh, Q. Rev. Biophys., 18 (1985) 323-422.

[10] M. W. Washabaugh and K. D. Collins, J. Biol. Chem., 261 (1986) 2477-2485.

[11] K. D. Collins, Proc. Natl Acad. Sci. USA, 92 (1995) 5553-5557.

[12] K. D. Collins, Methods, 34 (2004) 300-311.

[13] R. Bartucci and L. Sportelli, Biochim. Biophys. Acta, 1195 (1994) 229-236.

[14] R. Bartucci, S. Belsito and L. Sportelli, Chem. Phys. Lipids, 79 (1996) 171-180. 
[15] R. Paweloszek, S. Briançon, Y. Chevalier, N. Gilon-Delepine, J. Pelletier and M.-A. Bolzinger, Pharm. Res., 33 (2016) 1576-1586.

[16] E. Michael-Baruch, Y. Shiri and S. Cohen, J. Pharm. Sci., 83 (1994) 1071-1076.

[17] Y. I. I. Ko, S. S. Kim and S. K. Han, Arch. Pharm. Res., 18 (1995) 231-236.

[18] M. Tarnowska, S. Briançon, J. Resende de Azevedo, Y. Chevalier, C. Barratier, T. Pourcher and M.-A. Bolzinger, Int. J. Cosmet. Sci., 41 (2019) 617-623.

[19] M. J. Bartek, J. A. LaBudde and H. I. Maibach, J. Invest. Dermatol. 58 (1972) 114-123.

[20] H. Brown, J. Biol. Chem., LXVIII (1926) 260.

[21] K. Berend, L. H. van Hulsteijn and R. O. B. Gans, Eur. J. Int. Med., 23 (2012) 203-211.

[22] T. J. Franz, J. Invest. Dermatol., 64 (1975) 190-195.

[23] M. Förster, M.-A. Bolzinger, M.-R. Rovère, O. Damour, G. Montagnac and S. Briançon, Skin Pharmacol. Physiol., 24 (2011) 103-112.

[24] OECD, Guidance Document for the Conduct of Skin Absorption Studies, 2004, vol. 2.

[25] H. Scheffé, J. R. Stat. Soc. Ser. B, 25 (1963) 235-251.

[26] J. Cornell, Experiments with Mixtures: Designs, Models, and the Analysis of Mixture Data, New York, $3^{\text {rd }}$ edn, 2002.

[27] R. H. Stokes and R. A. Robinson, J. Am. Chem. Soc., 70 (1948) 1870-1878.

[28] K. S. Pitzer, J. Phys. Chem. 77, (1973) 268-277.

[29] J. N. Israelachvili, Intermolecular \& Surface Forces, Academic Press, London, $2^{\text {nd }}$ edn, 1991, p. 55.

[30] M. J. Blandamer, J. B. F. N. Engberts, P. T. Gleeson and J. C. R. Reis, Chem. Soc. Rev., 34 (2005) 440-458.

[31] M. S. Roberts, Y. G. Anissimov and R. A. Gonsalvez, in R. L. Bronaugh and H. I. Maibach (Eds), Percutaneous Absorption Drugs-Cosmetics-Mechanisms-Methodology, Marcel Dekker, New York, $3^{\text {rd }}$ edn, 1999 , pp. 7-8.

[32] H. R. Elden, in H. R. Elden (Ed.), Biophysical Properties of the Skin, Wiley-Interscience, New York, 1971, pp. 115-123.

[33] E. Ruckenstein and M. Manciu, Adv. Colloid Interface Sci., 105 (2003) 63-101.

[34] A. Kalra, N. Tugcu, S. M. Cramer and S. Garde, J. Phys. Chem. B, 105 (2001) 6380-6386.

[35] B. W. Ninham and V. Yaminsky, Langmuir, 13 (2002) 2097-2108.

[36] K. Fajans, Naturwiss., 9 (1921) 729-738.

[37] D. F. C. Morris, Struct. Bond., 4 (1968) 63-82.

[38] K. D. Collins, Biophys. J., 72 (1997) 65-76.

[39] K. D. Collins, G. W. Neilson and J. E. Enderby, Biophys. Chem., 128 (2007) 95-104.

[40] D. F. Parsons, M. Boström, T. J. Maceina, A. Salis and B. W. Ninham, Langmuir, 26 (2010) 3323-3328.

[41] N. Schwierz, D. Horinek and R. R. Netz, Langmuir, 26 (2010) 7370-7379.

[42] L. Bertinetti, P. Fratzl and T. Zemb, New J. Phys., 18 (2016) 083048.

[43] T. Mauro, G. Bench, E. Sidderas-Haddad, K. Feingold, P. Elias and C. Cullander, J. Invest. Dermatol., 111 (1998) 1198-1201. 